\title{
Larvicidal activity of lignans and alkaloid identified in Zanthoxylum piperitum bark toward insecticide-susceptible and wild Culex pipiens pallens and Aedes aegypti
}

\author{
Soon-II Kim ${ }^{1}$ and Young-Joon Ahn ${ }^{2^{*}}$
}

\begin{abstract}
Background: The yellow fever mosquito, Aedes aegypti, and the common house mosquito, Culex pipiens pallens, transmit dengue fever and West Nile virus diseases, respectively. This study was conducted to determine the toxicity of the three lignans (-)-asarinin, sesamin and (+)-xanthoxylol-y,y-dimethylallylether (XDA), and the alkaloid pellitorine from Zanthoxylum piperitum (Rutaceae) bark to third-instar larvae from insecticide-susceptible C. pipiens pallens and Ae. aegypti as well as wild C. pipiens pallens resistant to deltamethrin, cyfluthrin, fenthion, and temephos.

Methods: The toxicities of all isolates were compared with those of mosquito larvicide temephos. $L C_{50}$ values for each species and their treatments were significantly different from one another when their $95 \%$ confidence intervals did not overlap.

Results: XDA was isolated from Z. piperitum as a new larvicidal principle. XDA ( $L C_{50}, 0.27$ and $\left.0.24 \mathrm{mg} / \mathrm{l}\right)$ was 4, 53, and 144 times and 4,100, and 117 times more toxic than pellitorine, sesamin, and asarinin toward larvae from susceptible C. pipiens pallens and Ae. aegypti, respectively. Overall, all the isolates were less toxic than temephos $\left(\mathrm{LC}_{50}, 0.006\right.$ and $\left.0.009 \mathrm{mg} / \mathrm{l}\right)$. These constituents did not differ in toxicity to larvae from the two Culex strains. The present finding indicates that the lignans and alkaloid and the insecticides do not share a common mode of larvicidal action or elicit cross-resistance.

Conclusion: Naturally occurring Z. piperitum bark-derived compounds, particularly XDA, merit further study as potential mosquito larval control agents or as lead compounds for the control of insecticide-resistant mosquito populations.
\end{abstract}

Keywords: Botanical mosquito larvicide, Zanthoxylum piperitum, Rutaceae, Lignans, Xanthoxylol-ү,Y-dimethylallylether, Alkaloid, Insecticide resistance

\section{Background}

The yellow fever mosquito, Aedes aegypti (Linnaeus, 1762) [1], and the common house mosquito, Culex pipiens pallens (Coquillett, 1898) [2], are found in tropical and subtropical regions of the world [3] and Eastern Asia [4], respectively, and are serious disease vectoring insect pests $[5,6]$. A recent study calculated that more than 2.5 billion people are at risk of dengue infection

\footnotetext{
* Correspondence: yjahn@snu.ac.kr

${ }^{2}$ Department of Agricultural Biotechnology, Seoul National University, Seoul 08826, South Korea

Full list of author information is available at the end of the article
}

over 100 countries worldwide, and there may be $50-100$ million dengue infections annually, including 22,000 deaths every year, mostly among children [7]. From 1999 to $2015,43,937$ cases of human West Nile virus disease (including 20,265 neuroinvasive disease cases) were reported in the United States (US), which resulted in 1,911 deaths [8]. The most serious problem with the mosquito species is their ability to evolve resistance to insecticides rapidly [9]. Increasing levels of resistance to the conventional insecticides have resulted in multiple treatments and excessive doses, raising serious environmental and human health concerns. Widespread insecticide resistance 
has been one of the major obstacles in the cost-effective integrated vector management program. In addition, the number of approved insecticides may be reduced soon in the US by the US Environmental Protection Agency as reregistration occurs [10]. Reregistration requirement is also a concern in other regions including in the European Union, where it is under the control of the Commission Regulation (EC) No 1048/2005 [11]. Therefore, there is a high need for the development of selective control alternatives with novel target sites to establish a biorational resistance management strategy based on all available information on the extent and nature of resistance in mosquitoes because vaccines have limited effectiveness in controlling dengue [12].

Biocides derived from plants have been suggested as potential alternatives for mosquito control largely because plants constitute a potential source of bioactive secondary metabolites that are perceived by the public as relatively safe and with less risk to the environment, and with minimal impacts to human and animal health [13-19]. Phytochemicals act at multiple, novel target sites [14, 16-21], thereby reducing the potential for resistance [17-19, 22, 23]. Based on these benefits of botanical insecticides, numerous papers are published annually [19, 24]. Phytochemicals are regarded as potential sources to develop commercial mosquito larvicides as products derived from certain plants and their constituents meet the criteria as reduced risk insecticides [16-19, 25]. Recently, Zanthoxylum plants (Rutaceae) have drawn attention because they contain insecticidal constituents toward the cowpea aphid, Aphis craccivora Koch, 1854 [26, 27], the maize weevil, Sitophilus zeamais (Motschulsky, 1855) $[28,29]$, and larvae of various mosquito vectors [17, 30]. However, no previous studies have investigated the potential use of Japanese pepper, Zanthoxylum piperitum (L.) DC., for managing mosquitoes, particularly insecticide-resistant mosquitoes, despite its repellency to Ae. aegypti [31] and the stable fly, Stomoxys calcitrans (Linnaeus, 1758) [32, 33].

In this study, our aim was to assess whether the three lignans, asarinin, xanthoxylol- $\gamma, \gamma$-dimethylallylether (XDA) and sesamin, and the isobutylamide alkaloid pellitorine, extracted from the bark of $Z$. piperitum, had the toxicity to third-instar larvae from insecticide-susceptible $C$. pipiens pallens and Ae. aegypti, as well as wild colonies of $C$. pipiens pallens resistant to various insecticides [23]. The toxicity of the bark constituents was compared with that of the currently available mosquito larvicide temephos to assess their use as future commercial mosquito larvicides because it is registered as a larvicide for the control of mosquitoes in South Korea [34]. Also, the quantitative structure-activity relationship (QSAR) of the test compounds is discussed.

\section{Methods}

\section{Instrumental analysis}

The ${ }^{1} \mathrm{H}$ and ${ }^{13} \mathrm{C}$ nuclear magnetic resonance (NMR) spectra were recorded in $\mathrm{CDCl}_{3}$ on Varian NMR system spectrometers (Varian, Palo Alto, CA, USA), using tetramethylsilane as an internal standard. The chemical shifts are given in $\delta(\mathrm{ppm})$. The ultraviolet (UV) spectra were obtained in methanol on a UVICON 933/934 spectrophotometer (Kontron, Milan, Italy) and the mass spectra on a GSX 400 spectrometer (Jeol, Tokyo, Japan). Silica gel 60 (0.063-0.2 mm) (Merck, Darmstadt, Germany) and Sephadex LH-20 (Sigma-Aldrich, St. Louis, MO, USA) were used for column chromatography. Merck precoated silica gel plates (Kieselgel $60 \mathrm{~F}_{254}$ ) were used for analytical thin-layer chromatography (TLC). An Agilent 1200 series high-performance liquid chromatograph (Agilent, Santa Clara, CA, USA) was used to isolate the active constituents.

\section{Materials}

The organophosphorus (OP) insecticide temephos (97.3\%) was purchased from Riedel (Seelze, Lower Saxony, Germany). Triton X-100 was purchased from Coseal (Seoul, South Korea). All of the other chemicals used in this study were of reagent-grade quality and are available commercially.

\section{Mosquitoes}

The stock cultures of $C$. pipiens pallens (susceptible KS-CP strain) and $A e$. aegypti have been maintained in the laboratory without exposure to any known insecticide, as described previously [35]. Larvae from YS-CP colony of C. pipiens pallens, originally collected near rice paddy fields and cowsheds in Yusung (Daejeon, South Korea) in September 2010, showed extremely high levels of resistance to fenthion (resistance ratio (RR), 390) and deltamethrin (RR, 164) and moderate levels of resistance to cyfluthrin (RR, 14) and temephos (RR, 14) [23]. Adult mosquitoes were maintained on a $10 \%$ sucrose solution and blood fed on live mice. Larvae were reared in plastic trays $(24 \times 35 \times 5 \mathrm{~cm})$ containing $0.5 \mathrm{~g}$ of sterilised diet (40-mesh chick chow powder/yeast, $4 / 1$ by weight). All stages were held at $27 \pm 1{ }^{\circ} \mathrm{C}, 65-75 \%$ relative humidity, and a 14:10 h light:dark cycle.

\section{Plant material}

Fresh bark of $Z$. piperitum was collected from the Southern Forest Resources Research Center (Jinju, Gyeongnam, South Korea), National Institute of Forest Science, in midAugust 2009. A certified botanical taxonomist was used to identify the plant. A voucher specimen (ZP-01) was deposited in the Research Institute of Agriculture and Life Sciences, College of Agriculture and Life Sciences, Seoul National University. 


\section{Extraction and isolation}

Air-dried bark (550 g) of Z. piperitum was pulverised, extracted with methanol $(3.3 \mathrm{~L})$ two times at room temperature for 2 days, and filtered. The combined filtrate was concentrated to dryness by rotary evaporation at $40{ }^{\circ} \mathrm{C}$ to yield approximately $70 \mathrm{~g}$ of a dark brownish sticky solid. The extract (20 g) was sequentially partitioned into hexane- $(6.4 \mathrm{~g})$, chloroform- (1.36 g), ethyl acetate- $(0.46 \mathrm{~g})$, and water-soluble $(11.78 \mathrm{~g})$ portions for the subsequent bioassays. This fractionation procedure was repeated three times. The organic solvent-soluble portions were concentrated under vacuum at $35^{\circ} \mathrm{C}$, and the water-soluble portion was freeze-dried. To isolate the active constituents, $10-50 \mathrm{mg} / \mathrm{l}$ of each $Z$. piperitum bark-derived fraction was tested in a mortality bioassay, as described by Perumalsamy et al. [22].

The hexane-soluble fraction (19.2 g) was the most biologically active fraction (Table 1 ) and was chromatographed on a $5.5 \times 70 \mathrm{~cm}$ silica gel $(500 \mathrm{~g})$ column by elution with a gradient of chloroform and methanol [(100:0 (2 l), 95:5 (1 l), 90:10 (2 l), 80:20 (1 l), 50:50 (1 l), and $0: 100(1.5 \mathrm{l})$ by volume] to provide 34 fractions (each approximately $250 \mathrm{ml}$ ) (Fig. 1). The column fractions were monitored by TLC on silica gel plates developed with a chloroform and methanol (9:1 by volume) mobile phase. Column fractions with similar $R_{\mathrm{f}}$ values on the TLC plates were pooled. The spots were detected by spraying the plate with $4 \% \mathrm{H}_{2} \mathrm{SO}_{4}$ and then heating on a hot plate. Active fractions $11-17(\mathrm{H} 3)$ were pooled and rechromatographed on a $5.5 \times 70 \mathrm{~cm}$ silica gel $(500 \mathrm{~g})$ column by elution with a gradient of hexane and ethylacetate [(90:10 (1 l), 80:20 (1 l), and 0:100 (1 l) by volume] and finally with 11 methanol to afford 16 fractions (each approximately $250 \mathrm{ml}$ ). The fractions were monitored by TLC on silica gel plates developed with a hexane and ethyl acetate (7:3 by volume) mobile phase. Active fractions 8-13 (H33) were pooled and crystallised during being dried by rotary evaporation at $35{ }^{\circ} \mathrm{C}$ to yield compound one (H331). The residual portion (H332) was isolated by Sephadex LH-20 column chromatography using a mobile phase of methanol. Two active fractions 4-11 (H3322) and 12-21 (H3323) were obtained. The H3322 fraction (4.09 g) was rechromatographed on a $5.5 \times 70 \mathrm{~cm}$ silica gel $(120 \mathrm{~g})$ column Separation was achieved with a gradient of hexane and acetone [80:20 (2 l), 70:30 (1 l), 50:50 (1 l), and 0:100 $(0.5 \mathrm{l})$ by volume] and finally with $1 \mathrm{l}$ methanol to afford 25 fractions (each approximately $200 \mathrm{ml}$ ). Column fractions were monitored by TLC on silica gel plates developed with a hexane and ethyl acetate (4:6 by volume) mobile phase. Active fractions 1-7 (H33221) were obtained. Fraction H33221 was rechromatographed on a silica gel column using a gradient of chloroform and ethyl acetate [20:1 (0.3 l), 10:1 (0.2 l), 8:2 (0.2 l), 1:1 $(0.1 \mathrm{l})$, and $0: 10(0.5 \mathrm{l})$ by volume] and finally with acetone $(0.2 \mathrm{l})$ to afford five fractions (each approximately $200 \mathrm{ml}$ ). A preparative high-performance liquid chromatography (HPLC) was performed to separate the constituents from the active H332212 fraction. The column was a $3.9 \mathrm{~mm}$ i.d. $\times 300 \mathrm{~mm}$ bondaclone ten silica (Phenomenex, Torrance, CA, USA) using a mobile phase of chloroform and ethyl acetate ( $95: 5$ by volume) at a flow rate of $1 \mathrm{ml} / \mathrm{min}$. Chromatographic separation was monitored using a UV detector at $264 \mathrm{~nm}$. The two active constituents two and three were isolated at retention times of 8.05 and $10.03 \mathrm{~min}$, respectively. For separation of a constituent from another active H3323 fraction (1.3 g), a preparative HPLC was performed. The column was a $21.2 \mathrm{~mm}$ i.d. $\times 250 \mathrm{~mm}$ Phenomenex Prodigy ODS with a mobile phase of acetonitrile and water (1:1 by volume) at a flow rate of $1 \mathrm{ml} / \mathrm{min}$. Chromatographic separation was monitored at $287 \mathrm{~nm}$. Finally, an active constituent four was isolated at a retention time of $5.35 \mathrm{~min}$.

\section{Bioassay}

A mortality bioassay [36] was used to assess the toxicity of all compounds to third-instar larvae from the susceptible and wild mosquitoes. In brief, each compound in acetone was suspended in distilled water with Triton X$100(20 \mu \mathrm{l} / \mathrm{l})$. Groups of 20 mosquito larvae were separately put into paper cups $(270 \mathrm{ml})$ containing each compound solution $(250 \mathrm{ml})$. Temephos served as a positive control and was similarly formulated. Negative

Table 1 Toxicity of fractions obtained from solvent partitioning of methanol extract of Zanthoxylum piperitum bark to third-instar larvae from Culex pipiens pallens during a $24 \mathrm{~h}$ exposure

\begin{tabular}{|c|c|c|c|c|c|c|}
\hline Material & $n^{a}$ & Slope \pm SE & $\mathrm{LC}_{50}, \mathrm{mg} / \mathrm{l}\left(95 \% \mathrm{Cl}^{\mathrm{b}}\right)$ & $\mathrm{LC}_{90}, \mathrm{mg} / \mathrm{l}\left(95 \% \mathrm{Cl}^{\mathrm{b}}\right)$ & $x^{2 c}$ & $P$-value \\
\hline Methanol extract & 240 & $5.1 \pm 0.57$ & $5.91(5.38-6.44)$ & $10.50(9.28-12.54)$ & 3.25 & 0.974 \\
\hline Hexane-soluble fraction & 240 & $4.3 \pm 0.47$ & $4.18(3.69-4.64)$ & 8.27 (7.26-9.89) & 4.90 & 0.932 \\
\hline Chloroform-soluble fraction & 240 & $5.1 \pm 0.54$ & $5.02(4.53-5.50)$ & $9.02(8.04-10.58)$ & 6.01 & 0.921 \\
\hline Ethyl acetate-soluble fraction & 60 & & $>100$ & & & \\
\hline Water-soluble fraction & 60 & & $>100$ & & & \\
\hline
\end{tabular}

${ }^{\mathrm{a}}$ Number of larvae tested

${ }^{\mathrm{b}} \mathrm{Cl}$ denotes confidence interval

'Pearson's chi-square goodness-of-fit test 


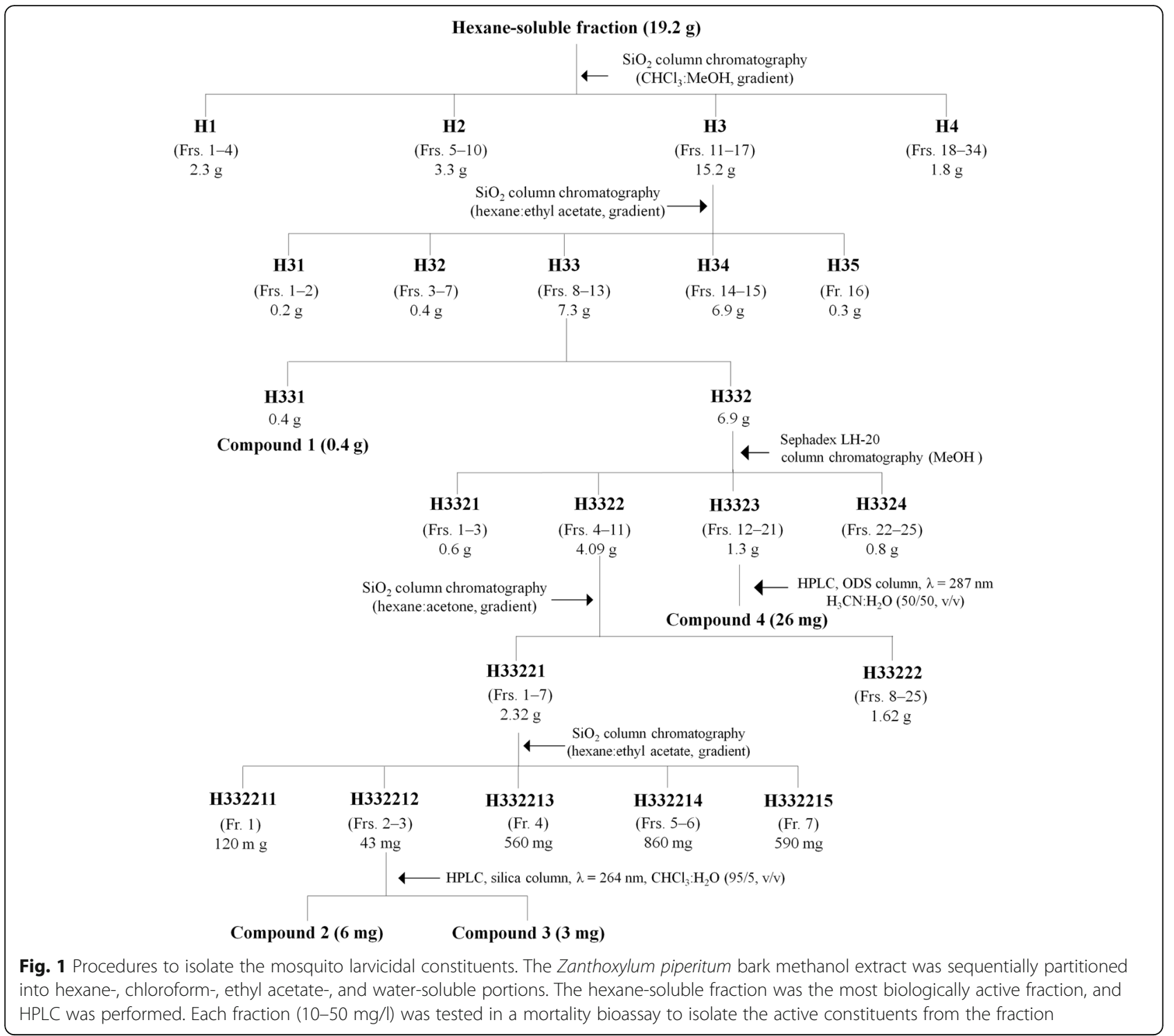

controls consisted of the acetone-Triton X-100 solution in distilled water. Based on the preliminary test results, the toxicity of each test compound and insecticide was determined with four to six concentrations ranging from 0.1 to $100 \mathrm{mg} / \mathrm{l}$ and 0.001 to $0.1 \mathrm{mg} / \mathrm{l}$, respectively. All treatments were replicated three times using 20 larvae per replicate.

Treated and control (acetone-Triton X-100 solution only) larvae were held under the same conditions as those used for colony maintenance without providing food. Larval mortalities were determined $24 \mathrm{~h}$ posttreatment. A larva was considered dead if it did not move when prodded with a fine wooden dowel [22].

\section{Data analysis}

Data were corrected for control mortality using Abbott's formula [37]. Concentration-mortality data were subjected to probit analysis [38]. A compound having $\mathrm{LC}_{50}>100 \mathrm{mg} / \mathrm{l}$ was ineffective as described by Kiran et al. [39]. The $\mathrm{LC}_{50}$ values for each species and their treatments were significantly different from one another when their 95\% confidence intervals did not overlap.

\section{Results}

Bioassay-guided fractionation and isolation

The fractions obtained from the solvent partitioning of the methanol extract of the $Z$. piperitum bark were bioassayed toward third-instar larvae from insecticidesusceptible C. pipiens pallens (Table 1) and Ae. aegypti (Table 2). Significant differences in toxicity were observed among the fractions and were used to identify the peak activity fractions for the next step of purification. Based on the $24 \mathrm{~h} \mathrm{LC} \mathrm{LC}_{50}$ values, the hexane-soluble fraction was the most toxic material, followed by the 
Table 2 Toxicity of fractions obtained from solvent partitioning of methanol extract of Zanthoxylum piperitum bark to third-instar larvae from Aedes aegypti during a $24 \mathrm{~h}$ exposure

\begin{tabular}{|c|c|c|c|c|c|c|}
\hline Material & $n^{a}$ & Slope \pm SE & $\mathrm{LC}_{50}, \mathrm{mg} / \mathrm{l}\left(95 \% \mathrm{Cl}^{\mathrm{b}}\right)$ & $\mathrm{LC}_{90}, \mathrm{mg} / \mathrm{l}\left(95 \% \mathrm{Cl}^{\mathrm{b}}\right)$ & $x^{2 c}$ & $P$-value \\
\hline Methanol extract & 240 & $4.3 \pm 0.47$ & $3.95(3.47-4.40)$ & $7.88(6.92-9.40)$ & 4.03 & 0.945 \\
\hline Hexane-soluble fraction & 240 & $4.0 \pm 0.43$ & $4.21(3.75-4.73)$ & $8.78(7.47-11.19)$ & 3.90 & 0.951 \\
\hline Chloroform-soluble fraction & 240 & $3.8 \pm 0.47$ & $5.68(5.06-6.33)$ & 12.30 (10.37-15.94) & 4.14 & 0.941 \\
\hline Ethyl acetate-soluble fraction & 60 & & $>100$ & & & \\
\hline Water-soluble fraction & 60 & & $>100$ & & & \\
\hline
\end{tabular}

${ }^{a}$ Number of larvae tested

${ }^{\mathrm{b}} \mathrm{Cl}$ denotes confidence interval

'Pearson's chi-square goodness-of-fit test

chloroform-soluble fraction. No toxicity was obtained using the ethyl acetate- or water-soluble fractions. Mortality in the acetone-Triton X-100-water-treated controls for any the species in this study was less than $2 \%$.

Bioassay-guided fractionation of the $Z$. piperitum bark extract afforded four active compounds that were identified by spectroscopic analyses, including electron ionized mass spectrometry (EI-MS) and NMR spectroscopy. The four active compounds were (-)-asarinin (5[3-(1,3-benzodioxol-5-yl)-1,3,3a,4,6,6a-hexahydrofuro[3, 4-c]furan-6-yl]-1,3-benzodioxole) (1), (+)-xanthoxylol$\gamma, \gamma$-dimethylallylether (XDA) (2), pellitorine $[(2 E, 4 E)-N$ (2-methylpropyl)deca-2,4-dienamide] (3), and sesamin [5, $5^{\prime}$ - $(1 S, 3 \mathrm{a} R, 4 S, 6 \mathrm{a} R)$-tetrahydro- $1 H, 3 H$-furo[3,4-c] furan-1 ,4-diylbis(1,3-benzodioxole)] (4) (Fig. 2). (-)-Asarinin (1) was identified based on the following evidence: white powder. EI-MS $(70 \mathrm{eV}), \mathrm{m} / z$ (\% relative intensity): 354 $[\mathrm{M}]^{+}, 336,203,161,149,135,122$ (Additional file 1). ${ }^{1} \mathrm{H}$ NMR $\left(\mathrm{CDCl}_{3}, 500 \mathrm{MHz}\right): \delta 2.85(1 \mathrm{H}, \mathrm{dd}, J=7.0,14.0 \mathrm{~Hz})$, $3.30(1 \mathrm{H}, \mathrm{m}), 3.83(2 \mathrm{H}, \mathrm{m}), 3.83-4.09(2 \mathrm{H}, \mathrm{m}), 4.40$ $(1 \mathrm{H}, \mathrm{d}, J=9.5 \mathrm{~Hz}), 4.82(1 \mathrm{H}, \mathrm{d}, J=7.0 \mathrm{~Hz}), 5.95(4 \mathrm{H}, \mathrm{d}$, $J=6.0 \mathrm{~Hz}), 6.79(4 \mathrm{H}, \mathrm{m}), 6.86(2 \mathrm{H}, \mathrm{s})$ (Additional file 2). ${ }^{13} \mathrm{C}$ NMR $\left(\mathrm{CDCl}_{3}, 125 \mathrm{MHz}\right): \delta 50.1 \mathrm{t}, 54.6 \mathrm{t}, 69.6 \mathrm{~d}, 70.9$ d, 82.0 d, 87.6 d, 100.9 t, 101.0 t, 106.3 d, 106.5 d, 108.1 d, $118.6 \mathrm{~d}, 119.5 \mathrm{~d}, 132.2 \mathrm{~s}, 135.1 \mathrm{~s}, 146.5 \mathrm{~s}, 147.1 \mathrm{~s}$, 147.6 s, 147.9 s (Additional file 3). (+)-Xanthoxylol- $\gamma, \gamma$-dimethylallylether (2) was characterized as follows: viscous solid. EI-MS $(70 \mathrm{eV}), \mathrm{m} / z$ (\% relative intensity): $424[\mathrm{M}]^{+}, 356$ (100), 325, 205, 178, 149, 135, 69 (Additional file 4). ${ }^{1} \mathrm{H}$ NMR $\left(\mathrm{CDCl}_{3}, 600 \mathrm{MHz}\right): \delta$ $1.74(3 \mathrm{H}, \mathrm{s}), 1.78(3 \mathrm{H}, \mathrm{s}), 2.92(1 \mathrm{H}, \mathrm{q}), 3.34(1 \mathrm{H}, \mathrm{m})$, $3.86(3 \mathrm{H}, \mathrm{m}), 3.88(3 \mathrm{H}, \mathrm{s}), 4.12(1 \mathrm{H}, \mathrm{d}, J=9.0 \mathrm{~Hz})$, $4.42(1 \mathrm{H}, \mathrm{d}, J=6.6 \mathrm{~Hz}), 4.59(2 \mathrm{H}, \mathrm{d}, J=6.6 \mathrm{~Hz}), 4.85$ $(1 \mathrm{H}, \mathrm{d}, J=5.4 \mathrm{~Hz}), 5.53(1 \mathrm{H}, \mathrm{m}), 5.97(2 \mathrm{H}, \mathrm{s}), 6.78$ $(1 \mathrm{H}, \mathrm{m}), 6.81(1 \mathrm{H}, \mathrm{m}), 6.84(1 \mathrm{H}, \mathrm{m}), 6.86(1 \mathrm{H}, \mathrm{m})$, $6.87(1 \mathrm{H}, \mathrm{m}), 6.92(1 \mathrm{H}, \mathrm{d}, J=1.2 \mathrm{~Hz})$ (Additional file 5). ${ }^{13} \mathrm{C}$ NMR $\left(\mathrm{CDCl}_{3}, 150 \mathrm{MHz}\right): \delta 18.4 \mathrm{q}, 26.0 \mathrm{q}, 50.4 \mathrm{~d}, 54.7$ d, 56.1 t, 66.1 t, 69.9 d, 71.2 d, 82.3 d, 87.9 d, 101.2 q, 106.6 d, 108.4 d, 109.6 d, 113.2 d, 118.6 d, 118.9 d, 120.2 d, 132.5 s, 133.8 s, 137.8 s, 146.8 s, 147.5 s, 147.9 s, 149.9 s (Additional file 6). Pellitorine (3) was characterized as follows: viscous oil. EI-MS $(70 \mathrm{eV}), \mathrm{m} / z$ (\% relative intensity): $223[\mathrm{M}]^{+}, 208,180,167,152$ (100), 113, 96, 72 (Additional file 7$)$. ${ }^{1} \mathrm{H}$ NMR $\left(\mathrm{CDCl}_{3}, 400 \mathrm{MHz}\right)$ : ${ }^{1} \mathrm{H}$ NMR $\left(\mathrm{CDCl}_{3}, 400 \mathrm{MHz}\right): \delta 0.88(3 \mathrm{H}, \mathrm{s}), 0.91(3 \mathrm{H}$, s), $0.93(3 \mathrm{H}, \mathrm{s}), 1.28(4 \mathrm{H}, \mathrm{m}), 1.37(2 \mathrm{H}, \mathrm{m}), 1.76(1 \mathrm{H}$, m), $2.13(2 \mathrm{H}, \mathrm{dd}, J=7.0,13.8 \mathrm{~Hz}), 3.16(2 \mathrm{H}, \mathrm{t}, J=6.4$, $12.9 \mathrm{~Hz}), 5.60(1 \mathrm{H}$, br s $), 5.76(1 \mathrm{H}, \mathrm{d}, J=15.0 \mathrm{~Hz})$, $6.09(2 \mathrm{H}, \mathrm{m}), 7.19(1 \mathrm{H}, \mathrm{d}, J=15.0 \mathrm{~Hz})$ (Additional file 8). ${ }^{13} \mathrm{C}$ NMR $\left(\mathrm{CDCl}_{3}, 100 \mathrm{MHz}\right): \delta 14.0 \mathrm{q}, 20.1 \mathrm{q}$, 22.5 t, 28.5 t, 28.6 d, 31.4 t, 32.9 t, 46.9 t, 121.7 d, $128.2 \mathrm{~d}, 128.2 \mathrm{~d}, 141.2 \mathrm{~d}, 166.4 \mathrm{~s}$ (Additional file 9).
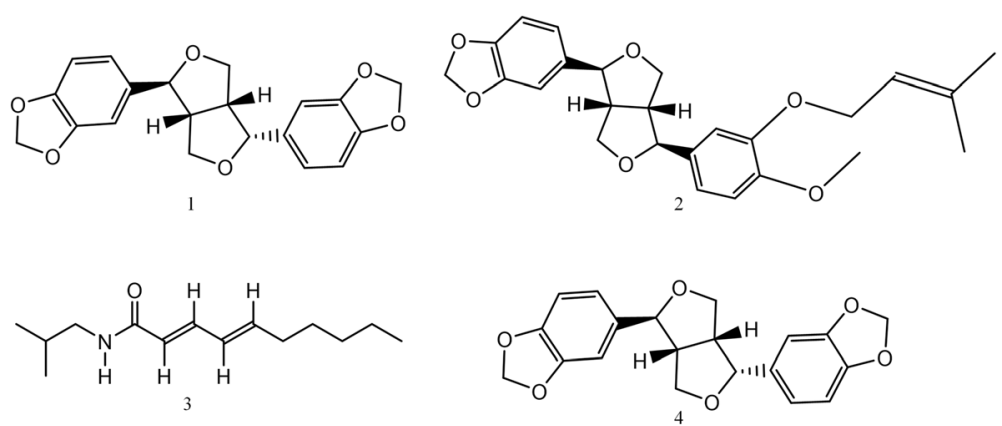

Fig. 2 Structures of asarinin, xanthoxylol-y,y-dimethylallylether, pellitorine, and sesamin. These compounds were identified in the bark of Zanthoxylum piperitum in this study. The chemical formula of (-)-asarinin (1) is $\mathrm{C}_{20} \mathrm{H}_{18} \mathrm{O}_{6}$, with a molar mass of $354.35 \mathrm{~g} / \mathrm{mol}$; the chemical formula of (+)-xanthoxylol- $\gamma, \gamma$-dimethylallylether (2) is $\mathrm{C}_{25} \mathrm{H}_{28} \mathrm{O}_{6}$, with a molar mass of $424.48 \mathrm{~g} / \mathrm{mol}$; the chemical formula of pellitorine (3) is $\mathrm{C}_{14} \mathrm{H}_{25} \mathrm{NO}$, with a molar mass of $223.35 \mathrm{~g} / \mathrm{mol}$; and the chemical formula of sesamin (4) is $\mathrm{C}_{20} \mathrm{H}_{18} \mathrm{O}_{6}$, with a molar mass of $354.35 \mathrm{~g} / \mathrm{mol}$ 
Sesamin (4) was characterized as follows: colorless crystals. EI-MS $(70 \mathrm{ev}), m / z$ (\% relative intensity): $354[\mathrm{M}]^{+}$, 323, 203, 178, 161, 149 (100), 135 (Additional file 10). ${ }^{1} \mathrm{H}$ NMR $\left(\mathrm{CDCl}_{3}, 500 \mathrm{MHz}\right): \delta 3.05(2 \mathrm{H}, \mathrm{m}), 3.86(2 \mathrm{H}, \mathrm{dd}, J$ $=3.0,9.0 \mathrm{~Hz}), 4.23(2 \mathrm{H}, \mathrm{dd}, J=6.5,9.0 \mathrm{~Hz}), 4.71(2 \mathrm{H}, \mathrm{d}, J$ $=4.0 \mathrm{~Hz}), 5.95(4 \mathrm{H}, \mathrm{s}), 6.79(4 \mathrm{H}, \mathrm{d}, J=8.0 \mathrm{~Hz}), 6.85(2 \mathrm{H}, \mathrm{s})$ (Additional file 11). ${ }^{13} \mathrm{C} \mathrm{NMR}\left(\mathrm{CDCl}_{3}, 125 \mathrm{MHz}\right): \delta 50.1 \mathrm{t}$, 54.6 t, 69.6 d, 70.9 d, 82.0 d, 87.6 d, 100.9 t, 101.0 t, 106.3 d, 106.5 d, 108.1 d, 118.6 d, 119.5 d, 122.0 s, $132.2 \mathrm{~s}$, $146.5 \mathrm{~s}, 147.1 \mathrm{~s}, 147.6 \mathrm{~s}, 147.9 \mathrm{~s}$ (Additional file 12).

\section{Larvicidal activity of test compounds}

The toxicity of the four isolated constituents to thirdinstar larvae from KS-CP strain of C. pipiens pallens was likewise compared with that of temephos, which was used as a positive control (Table 3). Responses varied according to compound examined. Based on the $24 \mathrm{~h} \mathrm{LC}_{50}$ values, XDA $(0.27 \mathrm{mg} / \mathrm{l})$ was the most toxic compound, followed by pellitorine $(1.12 \mathrm{mg} / \mathrm{l})$. These constituents were 45 and 187 times less toxic than temephos, respectively. $\mathrm{LC}_{50}$ of sesamin was $14.28 \mathrm{mg} / \mathrm{l}$. The toxicity of asarinin was the lowest of any of the compounds examined. Interestingly, the toxicity of all constituents was virtually identical toward both insecticide-susceptible and wild third-instar $C$. pipiens pallens larvae (Table 4), indicating a lack of crossresistance in the resistant larvae.

Toward third-instar Ae. aegypti larvae (Table 5), XDA $\left(\mathrm{LC}_{50}, 0.24 \mathrm{mg} / \mathrm{l}\right)$ was the most toxic compound, followed by pellitorine $\left(\mathrm{LC}_{50}, 0.98 \mathrm{mg} / \mathrm{l}\right)$, as judged by the $24 \mathrm{~h}$ $\mathrm{LC}_{50}$ values. These constituents were 27 and 109 times less toxic than temephos, respectively. $\mathrm{LC}_{50}$ of sesamin and asarinin was 23.98 and $28.15 \mathrm{mg} / \mathrm{l}$, respectively.

\section{Discussion}

Certain plant-derived materials and their constituents can be developed into products suitable for integrated mosquito management because they are selective, biodegrade to nontoxic products, have few harmful effects on nontarget organisms, are environmentally nonpersistent, and can be used in conjunction with biological control $[13-19,40]$. These potential mosquito larvicides can be applied to mosquito breeding places in the same manner as conventional mosquito larvicides. Although several plant preparations meet the criteria for efficacy, only a very few commercial botanical products have been marketed because there are some formidable barriers to commercialization, as well described previously $[17-19,40]$. In addition, some botanical insecticides may contain nonselective substances that may have a negative impact on small nontarget organisms such as crustaceans and zooplankton $[17,40]$ and human (e.g. contact dermatitis, allergic reactions, severe acute poisoning) [40]. Many plant-derived products and their constituents manifest toxicity to different mosquito species larvae $[13,14,16,17,30]$, and have been proposed as alternatives to conventional mosquito larvicides. Komalamisra et al. [41] considered larvicidal products exerting $\mathrm{LC}_{50}<$ $50 \mathrm{mg} / \mathrm{l}$ active, $50 \mathrm{mg} / \mathrm{l}<\mathrm{LC}_{50}<100 \mathrm{mg} / \mathrm{l}$ moderately active, $100 \mathrm{mg} / \mathrm{l}<\mathrm{LC}_{50}<750 \mathrm{mg} / \mathrm{l}$ effective, and $\mathrm{LC}_{50}>$ $750 \mathrm{mg} / \mathrm{l}$ inactive. Kiran et al. [39] considered compounds with $\mathrm{LC}_{50}<100 \mathrm{mg} / \mathrm{l}$ as exhibiting a significant larvicidal effect. It has been reported that the most promising botanical mosquito control agents are plants in the families Asteraceae, Cladophoraceae, Lamiaceae, Meliaceae, Oocystaceae, and Rutaceae [13]. The efficacy of various plant extracts and their fractions $\left(\mathrm{LC}_{50}, 2.6-\right.$ $44,400 \mathrm{mg} / \mathrm{l})$ and essential oils $\left(\mathrm{LC}_{50}, 0.2-194 \mathrm{mg} / \mathrm{l}\right.$; $\mathrm{LC}_{90}, 0.5-260 \mathrm{mg} / \mathrm{l}$ ) toward various mosquito species larvae has been well documented by Shaalan et al. [14] and Pavela [17], respectively, although the larvicidal activity can vary significantly according to plant species, chemotypes, plant tissue, age of plant, geographic conditions, solvent used in extraction, and mosquito species $[13,17,40]$. In the current study, Z. piperitum (Rutaceae) bark methanol extract and its hexane- and chloroformsoluble fractions exhibited good larvicidal activity toward C. pipiens pallens $\left(\mathrm{LC}_{50}, 4.18-5.91 \mathrm{mg} / \mathrm{l} ; \mathrm{LC}_{90}, 8.27-\right.$ $10.50 \mathrm{mg} / \mathrm{l})$ and Ae aegypti ( $\mathrm{LC}_{50}, 3.95-5.68 \mathrm{mg} / \mathrm{l} ; \mathrm{LC}_{90}$, $7.88-12.30 \mathrm{mg} / \mathrm{l})$. Zanthoxylum piperitum is distributed in northeast Asia (Korea, China, and Japan) [42], and the Z. piperitum bark contains unsaturated aliphatic acid amides [43]. Pavela and Govindarajan [30] reported that the

Table 3 Toxicity of Zanthoxylum piperitum bark constituents and temephos to third-instar larvae from insecticide-susceptible KS-CP strain of Culex pipiens pallens during a $24 \mathrm{~h}$ exposure

\begin{tabular}{|c|c|c|c|c|c|c|}
\hline Compound & $n^{\mathrm{a}}$ & Slope \pm SE & $\mathrm{LC}_{50}, \mathrm{mg} / \mathrm{l}\left(95 \% \mathrm{Cl}^{\mathrm{b}}\right)$ & $\mathrm{LC}_{90}, \mathrm{mg} / \mathrm{l}\left(95 \% \mathrm{Cl}^{\mathrm{b}}\right)$ & $x^{2 c}$ & $P$-value \\
\hline$\overline{X D A}(2)^{d}$ & 420 & $1.8 \pm 0.10$ & $0.27(0.24-0.30)$ & $1.44(1.21-1.79)$ & 6.61 & 0.980 \\
\hline Pellitorine (3) & 300 & $2.5 \pm 0.23$ & $1.12(0.95-1.35)$ & $3.75(2.95-5.20)$ & 5.67 & 0.957 \\
\hline Sesamin (4) & 240 & $2.8 \pm 0.31$ & $14.28(12.24-16.66)$ & $40.48(32.03-56.93)$ & 1.82 & 0.997 \\
\hline Asarinin (1) & 300 & $4.4 \pm 0.45$ & $38.90(35.26-42.50)$ & 75.77 (67.01-89.69) & 6.34 & 0.932 \\
\hline Temephos & 300 & $1.4 \pm 0.18$ & $0.006(0.005-0.008)$ & $0.049(0.032-0.096)$ & 2.49 & 0.999 \\
\hline
\end{tabular}

${ }^{\mathrm{a}}$ Number of larvae tested

${ }^{\mathrm{b}} \mathrm{Cl}$ denotes confidence interval

'Pearson's chi-square goodness-of-fit test

${ }^{d}$ Xanthoxylol- $\gamma, \gamma$-dimethylallylether 
Table 4 Toxicity of Zanthoxylum piperitum bark constituents and temephos to third-instar larvae from wild YS-CP colony of Culex pipiens pallens during a $24 \mathrm{~h}$ exposure

\begin{tabular}{|c|c|c|c|c|c|c|}
\hline Compound & $n^{\mathrm{a}}$ & Slope \pm SE & $\mathrm{LC}_{50}, \mathrm{mg} / \mathrm{l}\left(95 \% \mathrm{Cl}^{\mathrm{b}}\right)$ & $\mathrm{LC}_{90}, \mathrm{mg} / \mathrm{l}\left(95 \% \mathrm{Cl}^{\mathrm{b}}\right)$ & $x^{2 c}$ & $P$-value \\
\hline $\mathrm{XDA}(2)^{d}$ & 300 & $1.9 \pm 0.21$ & $0.31(0.26-0.38)$ & $1.39(1.03-2.13)$ & 6.23 & 0.937 \\
\hline Pellitorine (3) & 300 & $2.1 \pm 0.29$ & $1.42(1.17-1.80)$ & $5.46(3.71-10.29)$ & 1.81 & 0.997 \\
\hline Sesamin (4) & 300 & $2.3 \pm 0.24$ & $12.64(10.48-14.90)$ & $45.73(36.14-63.54)$ & 5.96 & 0.947 \\
\hline Asarinin (1) & 240 & $5.5 \pm 0.67$ & $33.80(31.30-36.47)$ & $57.67(51.01-69.59)$ & 4.41 & 0.927 \\
\hline Temephos & 360 & $4.1 \pm 0.34$ & $0.149(0.133-0.166)$ & $0.307(0.271-0.358)$ & 6.90 & 0.975 \\
\hline
\end{tabular}

${ }^{a}$ Number of larvae tested

${ }^{\mathrm{b}} \mathrm{Cl}$ denotes confidence interval

'Pearson's chi-square goodness-of-fit test

${ }^{\mathrm{d} X a n t h o x y l o l-} \gamma, \gamma$-dimethylallylether

Zanthoxylum monophyllum leaf essential oil had potent larvicidal activity toward Anopheles subpictus ( $\mathrm{LC}_{50}$ and $\mathrm{LC}_{90}, 41.50$ and $\left.82.19 \mathrm{mg} / \mathrm{l}\right)$, Aedes albopictus ( $\mathrm{LC}_{50}$ and $\mathrm{LC}_{90}, 45.35$ and $88.07 \mathrm{mg} / \mathrm{l}$ ), and Culex tritaeniorhynchus ( $\mathrm{LC}_{50}$ and $\mathrm{LC}_{90}, 49.01$ and $92.08 \mathrm{mg} / \mathrm{l}$ ).

Phytochemicals such as alkaloids, phenols and terpenoids, alone or in combination, contribute to acute toxicity toward various arthropod species [15]. Active larvicidal constituents $\left(\mathrm{LC}_{50}<50 \mathrm{mg} / \mathrm{l}\right)$ [41] derived from plants include alkaloids (e.g. pellitorine, guineensine, pipercide, and retrofractamide $\mathrm{A}, \mathrm{LC}_{50} 0.004-0.86 \mathrm{mg} / \mathrm{l}$ [44]), terpenoids (e.g. quassin, $\mathrm{LC}_{50} 6.0 \mathrm{mg} / \mathrm{l}$ [45]; germacrene $\mathrm{D}-4$-ol and $\alpha$-cadinol, $\mathrm{LC}_{50} 6.12-7.26$ and $10.27-12.28 \mathrm{mg} / \mathrm{l}$ [30]), coumarins (e.g. imperatorin and osthole, $\mathrm{LC}_{50}$ 2.88 and $3.14 \mathrm{mg} / \mathrm{l}$ [23]), flavonoids (e.g. karanjin, karanjachromene, pongamol, and pongarotene, $\mathrm{LC}_{50}$ 14.61$37.61 \mathrm{mg} / \mathrm{l} \mathrm{[46]),} \mathrm{phenylpropanoids} \mathrm{(e.g.} \mathrm{methyleu-}$ genol and $\alpha$-asarone, $\mathrm{LC}_{50} 10.49$ and $26.99 \mathrm{mg} / \mathrm{l}$ [22]; ethyl $p$-methoxycinnamate and ethyl cinnamate, $\mathrm{LC}_{50}$ 12.3 and $24.1 \mathrm{mg} / \mathrm{l}$ [36]), neolignans (e.g. conocarpan, eupomatenoid-5, and eupomatenoid- $6, \mathrm{LC}_{50}<10 \mathrm{mg} / \mathrm{l}$ [47]), cyanogenic glycosides (e.g. dhurrin, $\mathrm{LC}_{50}$ $1.12 \mathrm{mg} / \mathrm{l}$ [48]), lactones (e.g. goniothalmin, $\mathrm{LC}_{50}$ 0.87-25.95 mg/l [49]), acetylenic alcohols (e.g. falcarinol and falcarindiol, $\mathrm{LC}_{50} 3.49$ and $6.51 \mathrm{mg} / \mathrm{l}$ [50]), phenols (e.g. 4-butoxymethylphenol, $\mathrm{LC}_{50} 0.05 \mathrm{mg} / \mathrm{l}$ [51]), and fatty acids (e.g. oleic acid and palmitic acid, $18.07-18.45$ and $34.50-42.96 \mathrm{mg} / \mathrm{l}[46])$.
In the current study, we used a mortality bioassay to identify the larvicidal constituents from the $Z$. piperitum bark extracts. The active constituents were determined to be the furofuranoid lignans (-)-asarinin (1), (+)-XDA (2) and sesamin (4), and the isobutylamide alkaloid pellitorine (3). The interpretations of the proton and carbon signals of compounds 1, 2, 3, and 4 were largely consistent with those of Perumalsamy et al. [22], Biavatti et al. [52], Perumalsamy et al. [22] and Park et al. [44], and Ju et al. [53], respectively. XDA was isolated from Z piperitum as a new larvicidal constituent. This compound was most toxic toward larvae of two vector mosquito species, although it was less toxic than temephos. Pellitorine was also highly toxic toward C. pipiens pallens and Ae. aegypti, as described previously [22, 45]. In addition, these constituents were also effective toward $C$. pipiens pallens larvae resistant to various insecticides. The present finding indicates that $Z$. piperitum bark-derived preparations containing the active constituents, particularly XDA and pellitorine, hold promise for the development of novel, effective, naturally occurring mosquito larvicides even toward currently insecticide-resistant mosquito populations, because XDA ( $\mathrm{LC}_{50}$ 0.24-0.27 mg/l for two mosquito species) and pellitorine ( $\mathrm{LC}_{50} 0.98-1.12 \mathrm{mg} / \mathrm{l}$ for two mosquito species) meet the stage 3 criteria $\left(\mathrm{LC}_{50}<1 \mathrm{mg} / \mathrm{l}\right)$ set by Shaalan et al. [14]. The next step stage 4 involves the determination of effective field application rates of various formulations in simulated field trials and/or small-scale field trials [14].

Table 5 Toxicity of Zanthoxylum piperitum bark constituents and temephos to third-instar larvae of Aedes aegypti during a $24 \mathrm{~h}$ exposure

\begin{tabular}{|c|c|c|c|c|c|c|}
\hline Compound & $n^{\mathrm{a}}$ & Slope \pm SE & $\mathrm{LC}_{50}, \mathrm{mg} / \mathrm{l}\left(95 \% \mathrm{Cl}^{\mathrm{b}}\right)$ & $\mathrm{LC}_{90}, \mathrm{mg} / \mathrm{l}\left(95 \% \mathrm{Cl}^{\mathrm{b}}\right)$ & $x^{2 c}$ & $P$-value \\
\hline $\mathrm{XDA}(2)^{\mathrm{d}}$ & 300 & $1.8 \pm 0.17$ & $0.24(0.20-0.30)$ & $1.29(0.95-1.97)$ & 6.37 & 0.983 \\
\hline Pellitorine (3) & 240 & $2.6 \pm 0.30$ & $0.98(0.84-1.16)$ & $2.98(2.30-4.36)$ & 3.49 & 0.967 \\
\hline Sesamin (4) & 240 & $2.4 \pm 0.25$ & $23.98(20.48-28.33)$ & $82.75(63.03-122.72)$ & 2.66 & 0.998 \\
\hline Asarinin (1) & 300 & $7.5 \pm 0.79$ & $28.15(26.25-29.95)$ & $41.60(38.51-46.18)$ & 2.07 & 0.995 \\
\hline Temephos & 240 & $1.6 \pm 0.38$ & $0.009(0.007-0.012)$ & $0.062(0.032-0.373)$ & 1.48 & 0.999 \\
\hline
\end{tabular}

${ }^{\mathrm{a}}$ Number of larvae tested

${ }^{\mathrm{b}} \mathrm{Cl}$ denotes confidence interval

'Pearson's chi-square goodness-of-fit test

${ }^{\mathrm{d} X a n t h o x y l o l-} \gamma, \gamma$-dimethylallylether 
QSARs of phytochemicals in many insects have been well noted. For example, Wang et al. [23] studied the toxicity of six linear furanocoumarins including imperatorin and six simple coumarins including osthole. They reported that the chemical structure and alkoxy substitution and length of the alkoxyl side chain at the C8 position are essential for imparting toxicity. Park et al. [44] reported that the larvicidal activity toward three vector mosquito species was much more pronounced in compounds such as guineensine, pipercide, and retrofractamide A with an isobutylamine moiety than in one such as piperine without this moiety among the methylenedioxyphenyl (MDP)-containing compounds. In addition, the isobutylamides with an MDP moiety was more active than the ones without an MDP moiety. The MDP moiety is thought to stabilise the chemical structure [54]. In the current study, XDA with an MDP moiety was more toxic than either asarinin or sesamin with two MDP moieties. In addition, sesamin was more toxic than asarinin, 7-epimer of sesamin. Our findings, along with previous studies, indicate that other factor(s) such as chemical structure, functional group, and isomerism, as well as hydrophobic $(\log P)$ and molecular refraction parameters, may play, in part, a role in determining the lignan toxicities to mosquito larvae, although the MDP moiety might contribute, to some extent, to the larvicidal effect.

An investigation of the modes of action and the resistance mechanisms of biolarvicides may contribute to the development of selective mosquito control alternatives with novel target sites. Major mechanisms of resistance to insecticides currently available to control mosquitoes are target site insensitivity that reduces sodium channel sensitivity to pyrethroid insecticides or sensitivity of acetylcholinesterase to OP and carbamate insecticides, as well as enhanced metabolism of various groups of insecticides $[55,56]$. Some phytochemicals were found to be highly effective toward insecticide-resistant mosquitoes $[14,22,23]$, and they are likely to be useful in resistance management strategies. For example, imperatorin and osthole are effective toward larvae from wild $C$. pipiens pallens with extremely high to moderate levels of resistance to cyfluthrin, deltamethrin, fenthion, and temephos [22]. The current findings that the three furofuranoid lignans and the isobutylamide alkaloid described were of equal toxicity to both insecticide-susceptible and -resistant larvae of $C$. pipiens pallens suggest that the phytochemicals and the pyrethroid and OP insecticides do not share a common mode of action or elicit cross-resistance. Detailed tests are needed to understand fully the exact mode of action of the furofuranoid lignans and the isobutylamide alkaloid, although the octopaminergic and $\gamma$-aminobutyric acid receptors have been suggested as novel target sites for some monoterpenoid essential oil constituents in the American cockroach [57] and the cotton bollworm [20] and the fruit fly [21], respectively. It has also been reported that tannins and pellitorine primarily affect the midgut epithelium and secondarily affect the gastric caeca and the malpigian tubules in C. pipiens larvae [58] and Ae. aegypti larvae [59], respectively.

\section{Conclusion}

Zanthoxylum piperitum bark-derived products containing xanthoxylol- $\gamma, \gamma$-dimethylallylether and pellitorine could be useful as larvicides in the control of mosquito populations, particularly in the light of their activity toward insecticide-resistant mosquito larvae. Further research is needed on the practical applications of plant-derived preparations as novel mosquito larvicides to establish their safety profiles in humans, although $Z$. piperitum is commonly used as a spice and as a traditional medicinal plant [60,61]. In addition, their effects on nontarget aquatic organisms including larvivorous fishes, biological control agents for mosquitoes [62], and the aquatic environment need to be established. Lastly, detailed tests are needed to understand how to improve the larvicidal potency and stability of the compounds isolated from $Z$. piperitum for eventual commercial development.

\section{Additional files}

\begin{tabular}{|c|}
\hline $\begin{array}{l}\left.\text { Additional file 2: }{ }^{1} \mathrm{H} \text { NMR ( } \mathrm{CDCl}_{3}, 500 \mathrm{MHz}\right) \text { spectrum of (-)-asarinin (1). } \\
\text { TIF } 60 \mathrm{~kb})\end{array}$ \\
\hline $\begin{array}{l}\text { Additional file 3: }{ }^{13} \mathrm{C} \mathrm{NMR}\left(\mathrm{CDCl}_{3}, 125 \mathrm{MHz}\right) \text { spectrum of (-)-asarinin (1) } \\
\text { TIF } 62 \mathrm{~kb})\end{array}$ \\
\hline e 4: El-MS spectrum of (+)-xanthoxylol- $y, y$-dimethylallylether \\
\hline $\begin{array}{l}\text { I file 5: }{ }^{1} \mathrm{H} \text { NMR }\left(\mathrm{CDCl}_{3}, 600 \mathrm{MHz}\right) \text { spectrum of (+)-xanthoxylol- } \gamma, y \\
\text { lether (2). (TIF } 98 \mathrm{~kb})\end{array}$ \\
\hline $\begin{array}{l}\text { al file 6: }{ }^{13} \mathrm{C} \mathrm{NMR}\left(\mathrm{CDCl}_{3}, 150 \mathrm{MHz}\right) \text { spectrum of (+)-xanthoxylol-y,y } \\
\text { ylether (2). (TIF } 87 \mathrm{~kb})\end{array}$ \\
\hline file 7: El-MS spectrum of pellitorine (3). (TIF $80 \mathrm{~kb}$ ) \\
\hline $\begin{array}{l}\text { Additional file 8: }{ }^{1} \mathrm{H} \mathrm{NMR}\left(\mathrm{CDCl}_{3}, 400 \mathrm{MHz}\right) \text { spectrum of pellitorine (3). } \\
\text { TIF } 110 \mathrm{~kb})\end{array}$ \\
\hline $\begin{array}{l}\text { dditional file 9: }{ }^{13} \mathrm{C} \text { NMR }\left(\mathrm{CDCl}_{3}, 100 \mathrm{MHz}\right) \text { spectrum of pellitorine (3). } \\
\text { IF } 82 \mathrm{~kb})\end{array}$ \\
\hline Additional file 10: El-MS spectrum of sesamin (4). (TIF 63 kb) \\
\hline $\begin{array}{l}\text { ional file 11: }{ }^{1} \mathrm{H} \text { NMR }\left(\mathrm{CDCl}_{3}, 500 \mathrm{MHz}\right) \text { spectrum of sesamin (4). } \\
\mathrm{kb})\end{array}$ \\
\hline $5 \mathrm{MH}$ 7) snectrum of sesamin (4) \\
\hline
\end{tabular}

Abbreviations

El-MS: Electron ionized mass spectrometry; HPLC: High-performance liquid chromatography; MDP: Methylenedioxyphenyl; NMR: Nuclear magnetic resonance; OP: Organophosphorus; QSAR: Quantitative structure-activity relationship; RR: Resistance ratio; TLC: Thin-layer chromatography; UV: Ultra violet; XDA: (+)-xanthoxylol- $\gamma, \gamma$-dimethylallylether 


\section{Acknowledgements}

The authors thank Dr Haribalan Perumalsamy for reviewing this manuscript We also thank He Min Shin for her assistance with mosquito rearing.

\section{Funding}

This research was supported by the Research Institute of Agriculture and Life Sciences, College of Agriculture and Life Sciences, Seoul National University and BioGreen 21 Program (PJ007109), the Rural Development Administration, to Y.-J. Ahn.

\section{Availability of data and materials}

All data are disclosed in the text or in tables in the article. El-MS, ${ }^{1} \mathrm{H}$ NMR, and ${ }^{13} \mathrm{C}$ NMR spectra of compounds 1, 2, 3, and 4 are provided as Additional files 1, $2,3,4,5,6,7,8,9,10,11$ and 12 , respectively.

\section{Authors' contributions}

$Y$-JA conceived and designed the experiments. S-IK performed the experiments. S-IK and Y-JA analyzed the data. Y-JA wrote the paper. All authors read and approved the final manuscript.

\section{Competing interests}

The authors declare that they have no competing interests.

\section{Consent of publication}

Not applicable.

\section{Ethics approval and consent to participate}

Ethical approval was obtained from the Institutional Animal Care and Use Committees of Seoul National University for this study.

\section{Publisher's Note}

Springer Nature remains neutral with regard to jurisdictional claims in published maps and institutional affiliations.

\section{Author details}

${ }^{1}$ NARESO R\&D Center, Seoul National University Business Incubator, Suwon 16614, South Korea. ${ }^{2}$ Department of Agricultural Biotechnology, Seoul National University, Seoul 08826, South Korea.

Received: 19 January 2017 Accepted: 22 April 2017

Published online: 04 May 2017

\section{References}

1. Mattingly PF, Stone A, Knight KL. Culex aegypti Linnaeus, 1762 (Insecta, Diptera); proposed validation and interpretation under the plenary powers of the species so named. Z.N. (S.) 1216. Bull Zool Nomencl. 1962;19:208-19.

2. Coquillett DW. Report on a collection of Japanese Diptera, presented to the U. S. National Museum by the Imperial University of Tokyo. Proc US Nat Mus. 1898;21:301-40.

3. Brown JE, Evans BR, Zheng W, Obas V, Barrera-Martinez L, Egizi A, et al. Human impacts have shaped historical and recent evolution in Aedes aegypti, the dengue and yellow fever mosquito. Evolution. 2014;68:514-25.

4. Scott JG, Yoshimizu MH, Kasai S. Pyrethroid resistance in Culex pipiens mosquitoes. Pestic Biochem Physiol. 2015;120:68-76.

5. Becker N, Petrić D, Zgomba M, Boase C, Dahl C, Madon M, et al. Mosquitoes and their control. 2nd ed. New York: Springer; 2010. p. 25-42.

6. Musso D, Gubler DJ. Zika virus. Clin Microbiol Rev. 2016;29:487-524

7. Bhatt S, Gething PW, Brady OJ, Messina JP, Farlow AW, Moyes CL, et al. The global distribution and burden of dengue. Nature. 2013:496:504-7.

8. Centers for Disease Control and Prevention: West Nile virus - statistics \& maps. 2016. https:/www.cdc.gov/westnile/statsmaps/. Accessed 15 Jan 2017.

9. Mota-Sanchez D, Whalon ME, Hollingworth RM, Xue Q. Documentation of pesticide resistance in arthropods. In: Whalon ME, Mota-Sanchez D, Hollingworth RM, editors. Global pesticide resistance in arthropods. Oxfordshire: CABl; 2008. p. 32-9.

10. Environmental Protection Agency: Pesticide reregistration performance measures and goals 1997-2008. 2016. https://www.epa.gov/pesticidereevaluation/pesticide-reregistration-performance-measures-and-goals-19972008. Accessed 15 Jan 2017.

11. European Union. Commission Regulation (EC) No. 1048/2005 of 13 June 2005 amending Regulation (EC) No. 2032/2003 on the second phase of the 10-year work programme referred to in Article 16(2) of Directive 98/8/EC of the European Parliament and of the Council concerning the placing of biocidal products on the market. Off J Eur Union. 2005;9:7.

12. Mahoney R. The introduction of new vaccines into developing countries. V: Will we lose a decade or more in the introduction of dengue vaccines to developing countries? Vaccine. 2014;32:904-8.

13. Sukumar K, Perich MJ, Boobar LR. Botanical derivatives in mosquito control: a review. J Am Mosq Control Assoc. 1991;7:210-37.

14. Shaalan E, Canyon D, Younes MWF, Abdel-Wahab H, Mansour A. A review of botanical phytochemicals with mosquitocidal potential. Environ Int. 2005;31: 1149-66.

15. Isman MB. Botanical insecticides, deterrents, and repellents in modern agriculture and an increasingly regulated world. Annu Rev Entomol. 2006;1:45-66.

16. Koul O, Walia S, Dhaliwal GS. Essential oils as green pesticides: potential and constraints. Biopestic Int. 2008:4:63-84.

17. Pavela R. Essential oils for the development of eco-friendly mosquito larvicides: a review. Ind Crops Prod. 2015:76:174-87.

18. Pavela R. History, presence and perspective of using plant extracts as commercial botanical insecticides and farm products for protection against insects - a review. Plant Protect Sci. 2016;52:229-41.

19. Pavela R, Benelli G. Essential oils as ecofriendly biopesticides? Challenges and constraints. Trends Plant Sci. 2016;21:1000-7.

20. Kostyukovsky M, Rafaeli A, Gileadi C, Demchenko N, Shaaya E. Activation of octopaminergic receptors by essential oil constituents isolated from aromatic plants: possible mode of action against insect pests. Pest Manag Sci. 2002:58:1101-6.

21. Priestley CM, Williamson EM, Wafford KA, Sattelle DB. Thymol, a constituent of thyme essential oil, is a positive allosteric modulator of human GABA receptors and a homo-oligomeric GABA receptor from Drosophila melanogaster. Br J Pharmacol. 2003;140:1363-72.

22. Perumalsamy $\mathrm{H}$, Chang KS, Park C, Ahn YJ. Larvicidal activity of Asarum heterotropoides root constituents against insecticide-susceptible and -resistant Culex pipiens pallens and Aedes aegypti and Ochlerotatus togoi. J Agric Food Chem. 2010:58:10001-6.

23. Wang Z, Kim JR, Wang M, Shu S, Ahn YJ. Larvicidal activity of Cnidium monnieri fruit coumarins and structurally related compounds against insecticide-susceptible and insecticide-resistant Culex pipiens pallens and Aedes aegypti. Pest Manag Sci. 2012:68:1041-7.

24. Isman MB, Grieneisen ML. Botanical insecticide research: many publications, limited useful data. Trends Plant Sci. 2014;19:140-5.

25. Isman MB. Botanical insecticides: for richer, for poorer. Pest Manag Sci. 2008;64:8-11.

26. Blackman RL, Eastop VF. Taxonomic issues. In: van Emden HF, Harrington R, editors. Aphids as crop pests. Oxfordshire: CABl; 2007. p. 1-29.

27. Nissanka AP, Karunaratne V, Bandara BM, Kumar V, Nakanishi T, Nishi M, et al. Antimicrobial alkaloids from Zanthoxylum tetraspermum and caudatum. Phytochemistry. 2001;56:857-61.

28. CABI. Sitophilus zeamais (greater grain weevil). Invasive Species Compendium. Oxfordshire: CABI; 2017. http://www.cabi.org/isc/datasheet/10926. Accessed 25 Apr 2017.

29. Wang CF, Yang K, Zhang HM, Cao J, Fang R, Liu ZL, et al. Components and insecticidal activity against the maize weevils of Zanthoxylum schinifolium fruits and leaves. Molecules. 2011:16:3077-88.

30. Pavela R, Govindarajan M. The essential oil from Zanthoxylum monophyllum a potential mosquito larvicide with low toxicity to the non-target fish Gambusia affinis. J Pest Sci. 2017;90:369-78.

31. Kamsuk K, Choochote W, Chaithong U, Jitpakdi A, Tippawangkosol P, Riyong D, et al. Effectiveness of Zanthoxylum piperitum-derived essential oil as an alternative repellent under laboratory and field applications. Parasitol Res. 2007;100:339-45.

32. Bishop FC. The stable fly (Stomoxys calcitrans L.), an important livestock pest. J Econ Entomol. 1913;6:112-26.

33. Hieu TT, Kim SI, Kwon HW, Ahn YJ. Enhanced repellency of binary mixtures of Zanthoxylum piperitum pericarp steam distillate or Zanthoxylum armatum seed oil constituents and Calophyllum inophyllum nut oil and their aerosols to Stomoxys calcitrans. Pest Manag Sci. 2010;66:1191-8.

34. Ministry of Food and Drug Safety: ezDrug database system. http://www. mfds.go.kr/index.do?x=0\&searchkey=title:contents\&mid=914\&searchword= \&pageNo=77. Accessed 13 Jan 2017.

35. Yang YC, Park IK, Kim EH, Lee HS, Ahn YJ. Larvicidal activity of medicinal plant extracts against Aedes aegypti, Aedes togoi, and Culex pipiens pallens (Diptera: Culicidae). J Asia-Pacific Entomol. 2004;7:227-32. 
36. Kim NJ, Byun SG, Cho JE, Chung K, Ahn YJ. Larvicidal activity of Kaempferia galanga rhizome phenylpropanoids towards three mosquito species. Pest Manag Sci. 2008;64:857-62.

37. Abbott WS. A method of computing the effectiveness of an insecticide. J Econ Entomol. 1925;18:265-7.

38. Institute SAS. SAS 9.13 program, 2nd ed. Cary: SAS Institute; 2014.

39. Kiran SR, Bhavani K, Devi PS, Rao BRR, Reddy KJ. Composition and larvicidal activity of leaves and stem essential oils of Chloroxylon swietenia DC against Aedes aegypti and Anopheles stephensi. Bioresour Technol. 2006;97:2481-4.

40. Masetti A. The potential use of essential oils against mosquito larvae: a short review. Bull Insectology. 2016;69:307-10.

41. Komalamisra N, Trongtokit $Y$, Rongsriyam $Y$, Apiwathnasorn C. Screening for larvicidal activity in some Thai plants against four mosquito vector species. Southeast Asian J TropMed Public Health. 2005;36:1412-22.

42. Seidemann J. World spice plants: economic usage, botany, taxonomy. New York: Springer; 2005.

43. Yasuda I, Takeya K, Itokawa H. Distribution of unsaturated aliphatic acid amides in Japanese Zanthoxylum species. Phytochemistry. 1982;21:1295-8.

44. Park IK, Lee SG, Shin SC, Park JD, Ahn YJ. Larvicidal activity of isobutylamides identified in Piper nigrum fruits against three mosquito species. J Agric Food Chem. 2002;50:1866-70.

45. Evans DA, Raj RK. Larvicidal efficacy of quassin against Culex quinquefasciatus. Indian J Med Res. 1991;93:324-7.

46. Perumalsamy $\mathrm{H}$, Jang MJ, Kim JR, Kadarkarai M, Ahn YJ. Larvicidal activity and possible mode of action of four flavonoids and two fatty acids identified in Millettia pinnata seed toward three mosquito species. Parasit Vectors. 2015;8:237.

47. Chauret DC, Bernard CB, Arnason JT, Durst T. Insecticidal neolignans from Piper decurrens. J Nat Prod. 1996;59:152-5.

48. Jackson FL, Behkeit SS, eL Etr SM, Quach NK. Larvicidal effects of grain sorghum (Sorghum bicolour) seedling extracts upon Culex pipiens larvae. J Am Mosq Control Assoc. 1990;6:500-3.

49. Kabir KE, Khan AR, Mosaddik MA. Goniothalamin - a potent mosquito larvicide from Bryonopsis laciniosa L. J Appl Entomol. 2003;127:112-5.

50. Eckenbach U, Lampman RL, Seigler DS, Ebinger J, Novak RJ. Mosquitocidal activity of acetylenic compounds from Cryptotaenia canadensis. J Chem Ecol. 1999;25:1885-93.

51. Sun R, Sacalis JN, Chin C, Still CC. Bioactive aromatic compounds from leaves and stems of Vanilla fragrans. J Agric Food Chem. 2001:49:5161-4.

52. Biavatti MW, Vieira PC, Silva FGF, Fernandes JB, Degani ALG, Cass OB, et al. Separation and NMR studies on lignans of Raulinoa echinata. Phytochem Anal. 2001;12:64-8.

53. Ju Y, Still CC, Sacalis JN, Li J, Ho CT. Cytotoxic coumarins and lignans from extracts of the northern prickly ash (Zanthoxylum americanum). Phytother Res. 2001;15:441-3.

54. Miyakado M, Nakayama I, Ohno N. Insecticidal unsaturated isobutylamides from natural products to agrochemical leads. In: Arnason JT, Philogène BJR, Morand P, editors. Insecticides of plant origin, ACS Symposium Series 387. Washington, DC: American Chemical Society; 1989. p. 173-87.

55. Hemingway J, Hawkes N, McCarroll L, Ranson H. The molecular basis of insecticide resistance in mosquitoes. Insect Biochem Mol Biol. 2004;34:653-65.

56. Li X, Schuler MA, Berenbaum MR. Molecular mechanisms of metabolic resistance to synthetic and natural xenobiotics. Annu Rev Entomol. 2007;52:231-53.

57. Enan E. Insecticidal activity of essential oils: octopaminergic sites of action. Comp Biochem Physiol C. 2001;130:325-37.

58. Rey D, Long A, Pautou MP, Meyran JC. Histopathological effects of tannic acid on the midgut epithelium of some aquatic Diptera larvae. J Invertebr Pathol. 1999;73:173-81

59. Perumalsamy H, Kim JR, Oh SM, Jung JW, Ahn YJ, Kwon HW. Novel histopathological and molecular effects of natural compound pellitorine on larval midgut epithelium and anal gills of Aedes aegypti. PLoS One. 2013;8:e80226

60. Perry LM. Medicinal plants of East and Southeast Asia: attributed properties and uses. Cambridge: MIT Press; 1980

61. Xie Z, Huang X. Dictionary of traditional Chinese medicine. Hong Kong: Commercial Press; 1984

62. Sharma VP, Ghosh A. Larvivorous fishes of inland ecosystems, Proceedings of the MRC-CIFRI Workshop. Delhi: India Malaria Research Centre (ICMR) publication; 1994

\section{Submit your next manuscript to BioMed Central and we will help you at every step:}

- We accept pre-submission inquiries

- Our selector tool helps you to find the most relevant journal

- We provide round the clock customer support

- Convenient online submission

- Thorough peer review

- Inclusion in PubMed and all major indexing services

- Maximum visibility for your research

Submit your manuscript at www.biomedcentral.com/submit 